\title{
Dermatology Life Quality Index and disease coping strategies in psoriasis patients
}

\author{
Sopiko Liluashvili ${ }^{1}$ Tina Kituashvili²
}

${ }^{1}$ Factury of Medicine, Department of Dermatology and Venereology, Ivane Javakhishvili Tbilisi State University, Tbilisi, Georgia ${ }^{2}$ Dermatology Department, National Center of Dermatology and Venereology, Tbilisi, Georgia

Adv Dermatol Allergol 2019; XXXVI (4): 419-424

DOI: https://doi.org/10.5114/ada.2018.75810

\begin{abstract}
Introduction: Psoriasis is a chronic, psycho-dermatological condition. Psychological factors can trigger and/or exacerbate skin lesion. Relapse of disease can be a source of stress and can worsen the patient's Quality of Life. Coping with psoriasis can create stress. This can become a vicious cycle.

Aim: To illustrate the connection between quality of life and coping strategies in a psoriasis patient group and to describe a methodology for identifying the risk group of patients who need immediate attention in terms of developing better coping strategies.

Material and methods: Fifty patients with psoriasis were enrolled in the study. The severity of psoriasis was assessed by the Psoriasis Area and Severity Index (PASI), the Dermatology Life Quality Index (DLQI) was used for quality of life assessment, and the COPE questionnaire was used for determination of coping strategies used by psoriasis patients.

Results: The results of the study indicate the relationship between quality of life and coping strategies. In patients with coping < 140 and at least elementary education, psoriasis disease is most likely to have an extremely large effect on quality of life as measured by the DLQI score being above 21.

Conclusions: Psoriasis is a distressing disorder that significantly impairs quality of life. There is a close link between quality of life and coping strategies. This study illustrates a methodology for identification of a risk group of patients with a highly decreased quality of life indicator and underdeveloped coping strategies. Future management of psoriasis may require involvement of multidisciplinary teams that help patients to manage the physical and psychological aspects of psoriasis.
\end{abstract}

Key words: psoriasis, Dermatology Life Quality Index, coping strategies.

\section{Introduction}

Psoriasis is a chronic, scaling inflammatory skin disease that affects approximately $1.5-2 \%$ of the world population [1]. Psoriasis has a significant negative impact on the patient's quality of life [2]. It has a detrimental effect on quality of life, which is comparable to that of ischemic disease, diabetes, depression and cancer [3]. Depending on the severity and location of outbreaks, individuals may experience significant physical discomfort and some disability [4]. It can give rise to negative reactions and avoidance of such patients by the social environment, ultimately affecting the mental state of the patient [5]. Psychological distress can lead to significant depression and social isolation; a high rate of suicidal ideation has been associated with psoriasis [6].

Psoriasis-related daily stress represents the chronic, recurrent, low-grade stress and hassles that occur largely as a result of having to live with a chronic, disfiguring disease. Stressful life events constitute a major risk of occurrence and recurrence, aggravating the severity of the disease and duration of symptoms. Anxiety and depression can worsen the condition or cause resistance to treatment.

Psychological distress is frequent among dermatological patients. The relationship between psychiatry and dermatology is often very complex [7]. Patients with psoriasis are focused more now than ever on finding solutions to these challenges, so they use coping strategies to invest own conscious effort, to solve personal and interpersonal problems in order to try to master, minimize or tolerate stress [8]. The effectiveness of the coping effort depends on the type of stress, the individual and circumstances. Maladaptive coping strategies can worsen the stress. There is similarity in the frequency of

Address for correspondence: Sopiko Liluashvili MD, Factury of Medicine, Department of Dermatology and Venereology, Ivane Javakhishvili Tbilisi State University, 8 Kartozia St, Tbilisi, Georgia, phone: +995 515 512340, e-mail: sl.liluashvili@gmail.com Received: 31.03.2018, accepted: 17.04.2018. 
use of specific coping strategies between patients with psoriasis and patients with other medical conditions, such as spinal cord injury, atrial fibrillation and cancer [9]. Greater mental stress in psoriasis patients is induced by stigma, which is more expressed in psoriasis patients compared with other skin diseases $[10,11]$.

\section{Aim}

While coping strategies and the psychological condition of psoriasis patients have been referenced by a number of studies, still no clear relationship between coping with psoriasis and quality of life has been identified. The aim of this study was to illustrate the connection between quality of life and coping strategies in the psoriasis patient group and to describe a methodology for identifying the risk group of patients that need immediate attention in terms of developing better coping strategies.

\section{Material and methods}

The study was conducted between February 2016 and November 2017. This was a questionnaire-based study of patients by attendance at a clinic at the Dermatology Department (National Centre of Dermatology and Venereology, Tbilisi, Georgia). The study received ethical approval from the local ethic committee.

All patients received printed information and provided their written informed consent for the management of personal data before participating in the study.

Inclusion criteria:

1. Aged 18 years old or over.

2. A clinical diagnosis of severe psoriasis confirmed by a dermatologist, including of psoriatic arthritis and nail involvement.
3. PASI score $>10$.

4. Formal consent to take part in the study. Exclusion criteria:

1. Age less than 18 years.

2. A diagnosis inconsistent with the clinical signs and symptoms of severe psoriasis, as well guttate, erythrodermic or pustular psoriasis.

3. Patients attending psychiatric consultation and treatment with psychotropic drugs.

The study included 50 patients with a diagnosis of severe psoriasis (PASI score >10). Patients were asked about their symptoms, to ensure they satisfied the clinical criteria for severe psoriasis. The following data were also captured at interview: current age, education, marital status, total duration of disease (years). All patients were assigned to the Caucasian ethnic group. In addition, patients were asked to confirm treatments received for their severe psoriasis (e.g. topical and oral therapies, phototherapy). Social and educational profile of the patients together with disease characteristics such as duration of disease and PASI score are presented in Table 1.

Further methods of patient analysis included:

1. Psoriasis Area and Severity Index (PASI); the most widely used tool to assess psoriasis severity in clinical trials, PASI reflects not only the body surface area, but also erythema, induration and scaling [12]. The total PASI score ranged from 0 to 72 points. Psoriasis was classified as severe when the PASI score was $>10$ points [13]. PASI score < 10 defines psoriasis as mild and may not be yet affecting the patient's quality of life in a sufficient way to reveal a connection with disease coping strategies [14].

2. Dermatology Life Quality Index (DLQI) - a validated Georgian version was used for quality of life assessment. The DLQI is a validated, 10-question, self-reported questionnaire to evaluate the patient's perception

Table 1. Social and educational profile of patients with psoriasis

\begin{tabular}{|c|c|c|c|}
\hline Variable & Total $(n=50)$ & Men $(n=25)$ & Women $(n=25)$ \\
\hline \multicolumn{4}{|l|}{ Marital status: } \\
\hline Single & 7 & 4 & 3 \\
\hline Married & 31 & 16 & 15 \\
\hline Divorced & 3 & 3 & 0 \\
\hline Widowed & 9 & 2 & 7 \\
\hline \multicolumn{4}{|l|}{ Education: } \\
\hline Elementary school & 7 & 3 & 4 \\
\hline High school & 6 & 4 & 2 \\
\hline Higher education & 37 & 18 & 19 \\
\hline Time of disease, mean \pm SD [years] & $15.94 \pm 13.94$ & $14.99 \pm 12.51$ & $16.89 \pm 15.45$ \\
\hline Age, mean \pm SD [years] & $47.88 \pm 14.63$ & $45.96 \pm 15.64$ & $49.8 \pm 13.58$ \\
\hline PASI score, mean \pm SD & $17.09 \pm 4.36$ & $16.53 \pm 3.6$ & $17.65 \pm 5.03$ \\
\hline
\end{tabular}


of the impact of psoriasis on quality of life $[15,16]$. The DLQI questionnaire was divided into 6 commonly identified categories. The DLQI was rated on a 4-point scale ( $0=$ not at all to $3=$ very much). The highest possible total score for the DLQI is 30 and higher scores indicate more severe impact on quality of life [17].

3. Coping Strategies Questionnaires (COPE) - which was developed to assess a broad range of coping responses. Like the DLQI questionnaire, the COPE was rated on a 4-point scale ( $1=$ I usually don't do this at all to $4=$ I usually do this a lot). Results from the Coping Strategies Questionnaire identified 15 different categories according to the complete version of COPE by Carver et al. [18].

\section{Statistical analysis}

Study analysis was conducted in the statistical programming language $\mathrm{R}$ and its integrated development environment RStudio. Supplementary materials contain all necessary data including $R$ codes that can easily be used to replicate the results of the study. Statistical methods used include Cronbach's $\alpha$ for measures of internal consistency of the questionnaire, Wilcoxon's test for hypothesis testing on paired-sample data, and finally, classification trees and regression analysis for predictions of DLQI scores based on the overall profile of patients and their strategies for coping with disease.

\section{Results}

Within the group of 50 patients participating in the study, 62\% experienced psoriasis having a very large effect on the quality of life and 32\% experienced it having an extremely large effect. A summary of effects on the patients' life and DLQI measures obtained from the questionnaire is presented in Figure 1.

A paired difference test was conducted for comparative analysis of results from questionnaires using Wilcoxon's test for matched pairs. For matching purposes, answers to the questionnaire on disease coping strategies were adapted to the same scale and relevance as measures of DLQI score (from lowest 0 to highest 3). Based on Wilcoxon's test results, the difference between the patients' overall score of DLQI and coping is less than 0.33 with a $p$-value of 0.04029 . This result suggests that patient coping is related to quality of life as measured by the DLQI score.

For further confidence regarding the reliability of the obtained results, internal consistency of questionnaires (DLQI and COPE) was computed by Cronbach's $\alpha$ measure. The reliability coefficient (standardized $\alpha$ ) based on the correlations resulted in $66 \%$. In addition, explained variance amounted to $77 \%$ as given by Guttman's $\lambda 6$ (G6) coefficient, while generally accepted cut-off rates for Cronbach's standardized $\alpha$ and Guttman's $\lambda$ coefficients are $60 \%$ and $70 \%$, respectively. Based on internal consistency and Wilcoxon's test results, it becomes further interesting to identify more specific relationships between quality of life within psoriasis patients and their overall profile characteristics as well as individual strategies for coping with disease.

A repeated process of binary recursive partitioning was used to identify the most relevant variables (characteristics of the patients) to be used in prediction of DLQI scores. As a result, classification (regression) trees were built as predictive models (decision trees) that result in DLQI score based on different characteristics of a patient. Decision trees were built to predict DLQI scores based on two types of data obtained in the study: the first type included the overall profile and total coping score of the patient; the second type included disease coping strategies. Resultant decision trees are illustrated in Figure 2.

Regression tree identified the following variables as relevant: "Coping score", "PASI score", "Age of disease", and "Marital status" for the first data type (Figure 2 A) and "Emotional and social support", "Mental health", "Positivity and growth", and "Planning" for the second data type (Figure 2 B).
Effect on patients' life

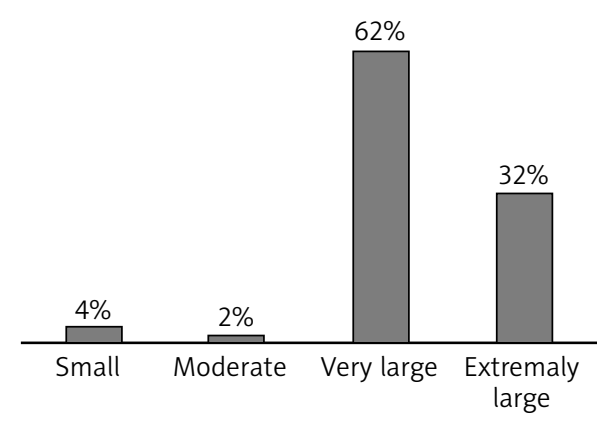

DQLI measures

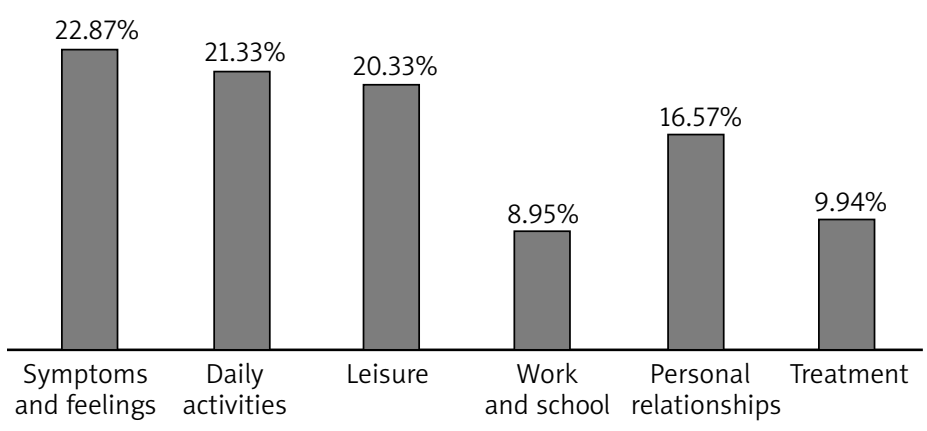

Figure 1. Summary of DLQI questionnaire results 

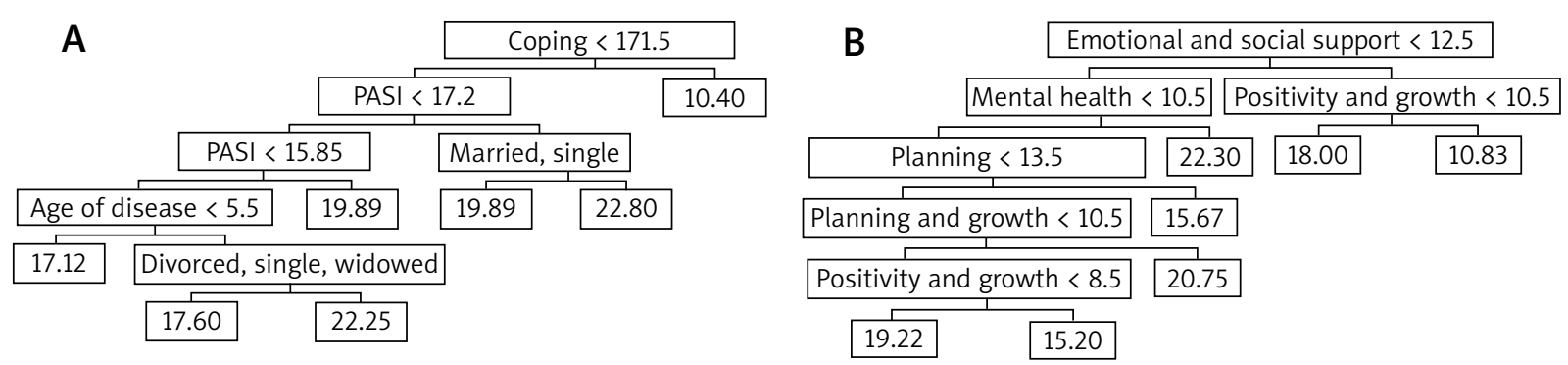

Figure 2. DLQI score predictions with decision trees
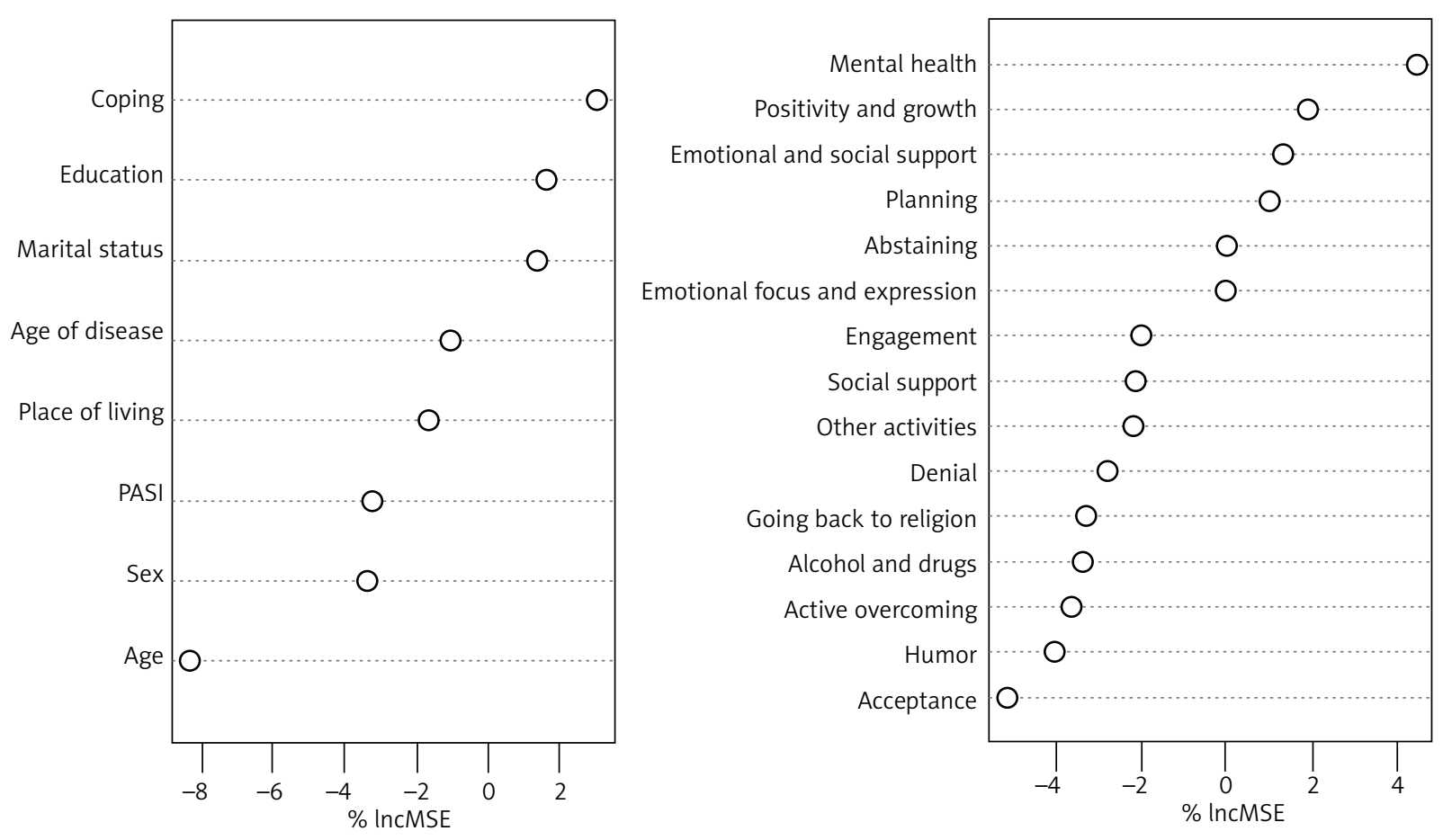

Figure 3. Random forest results for hierarchy of most explanatory variables in prediction of DLQI scores

In addition, Breiman's random forest algorithm was used to simulate 500 regression trees shown in Figure 2 (for both types of data). Random forest algorithm identified regression parameters that have the most explanatory power using a random sampling procedure and construction of a collection of decision trees with controlled variance. Explanatory power of a variable was measured by both the largest reduction in impurity and the smallest mean squared error (MSE) coefficient. Figure 3 illustrates the hierarchy of variables by their explanatory power.

It is suggested to use first three most explanatory variables to build regression models for prediction of DLQI scores. The random forest algorithm uses "Coping score", "Education" and "Marital status" from the first type of data set, and "Mental health", "Positivity and growth", and "Emotional and social support" from the second type of data set. Based on results from Figure 3, multiple regression analyses were conducted using dummy coefficients for categorical variables such as "Education" and "Marital status". A summary of the results from multiple regression on DLQI score as a dependent variable and independent variables from both types of data sets is given in Figure 4.

It is important to understand that overall coping score is negatively related to predicted DLQI score. Therefore, patients who employ coping strategies more are expected to receive a lower DLQI score and have better quality of life. Namely, overall coping score of a patient has regression coefficient of 0.07792 and a $p$-value of $8.08 \%$, followed by "Education" coefficients of around 32 and $p$-values very close to zero. Even though "Marital status" also has negative regression coefficients, its 
call:

$7 \mathrm{~m}($ formula $=$ DLQI $\sim$ coping + Education + Marital status - 1 , data $=$ DATA

Residuals:

Min 10 Median $3 Q \quad$ Max

$\begin{array}{lllll}-10.9740 & -2.6459 & 0.3935 & 3.5002 & 11.5785\end{array}$

coefficients:

Estimate std. Error $t$ value $\operatorname{Pr}(>|t|)$

Education Elementary $31.78445 \quad 7.10410 \quad 4.474 \quad 5.55 \mathrm{e}-05$ *中*

Education Higher $\quad 33.70066 \quad 6.66717 \quad 5.055 \quad 8.46 \mathrm{e}-06$ **

Education High $\quad 32.05031 \quad 6.67421 \quad 4.8021 .93 \mathrm{e}-05$ \#해

$\begin{array}{lllll}\text { Marital StatusMarried } & -4.31195 & 3.10905 & -1.387 & 0.1726\end{array}$

Marital statussingle $\quad-5.89032 \quad 3.66339 \quad-1.608 \quad 0.1152$

$\begin{array}{lllll}\text { Marital Statuswidowed } & -2.92682 & 3.49246 & -0.838 & 0.4066\end{array}$

---

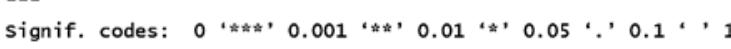

Residual standard error: 5.091 on 43 degrees of freedom

Multiple R-squared: 0.9371 , Adjusted R-squared: 0.9269

F-statistic: 91.58 on 7 and 43 DF, p-value: $<2.2 \mathrm{e}-16$ cal1:

$1 \mathrm{~m}($ formula $=$ DLQI $\sim$ Mental Health + Positivity and Growth +

Emotional and Social support - 1, data = DATA)

Residuals:

$$
\text { Min } 1 Q \text { Median } 3 Q \quad \text { Max }
$$

$\begin{array}{lllll}-17.3962 & -2.1241 & 0.6978 & 4.2319 & 13.7135\end{array}$ coefficients :

Estimate Std. Error $t$ value $\operatorname{Pr}(>|t|)$

$\begin{array}{llllll} & 0.46629 & 0.28414 & 1.641 & 0.107\end{array}$

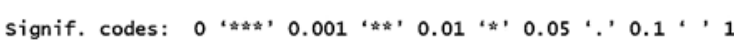

Residual standard error: 6.452 on 47 degrees of freedom

Multiple R-squared: 0.8897 , Adjusted R-squared: 0.8826

F-statistic: 126.3 on 3 and $47 \mathrm{DF}, \mathrm{p}$-value: $<2.2 \mathrm{e}-16$

Figure 4. Results from multiple regression analysis conducted on DLQI score prediction

p-values are above $11 \%$, and hence do not attain the commonly accepted level of statistical significance.

From the second data type, regression results showed that "Mental health" of the patient has the greatest significance in prediction of DLQI score with a coefficient of 1.35021 and a $p$-value being close to 0. Following "Mental health" is patient's "Positivity and growth" condition with a regression coefficient of 0.46629 and $p$-value of 10.7\%. Patient's "Emotional and social support" coefficient has very little effect (regression coefficient) in prediction of DLQI score with a $p$-value of $76.7 \%$ that does not suggest any statistical significance.

\section{Discussion}

The main goal of the research was to illustrate the connection between psoriasis patients' disease coping strategies and their quality of life. The relationship between these two measures determines psoriasis patients that experience greater negative effects on quality of life and hence require urgent attention to developing necessary coping skills.

The hypothesis that the average scores of disease coping strategies and quality of life are not significantly different was tested as a first step in developing the relationship between these two. With the $95 \%$ confidence interval on a scale from 0 to 3 the matched pair hypothesis test suggests that the population true difference is less than 0.33. This difference seems to be small on a scale from 0 to 3 but it still can be argued to be significant due to averaging, possible sampling errors, inefficient standardization, etc. It can also be argued that the hypothesis test result is not enough proof for concluding that there is a strong or any linear relationship between the two data sets.
Nevertheless, hypothesis testing concludes that average scores obtained from questionnaires on coping strategies and quality of life are close to each other. Now, it might be interesting to check how consistent the patients' responses to these questionnaires were - in other words, to check whether the responses follow similar patterns when compared to DLQI scores to understand whether patients responded adequately and sincerely to most of the questions with full understanding of their context.

Due to the categorical nature of questions an internal consistency test is an alternative to linear relationship measures such as correlation. One of the most widely used measures of internal consistency and reliability of the questionnaire is Cronbach's $\alpha$, which resulted in a consistency level above $65 \%$ and explained variance of the responses above $75 \%$. Cronbach's $\alpha$ for each category of the responses can be found in the supplementary materials. Satisfactory values indicated that the responses were adequate and consistent. This in turn enables us to continue the analysis of the linear relationship and to attempt to predict quality of life in patients based on their profile characteristics. Decision trees in Figure 2 illustrate an example of a prediction model. This model predicts that the patients with a coping score above 171 will have a DLQI score of around 10, thus qualifying them into the category of having a moderate effect on the quality of life. However, for patients with a coping score below 171 , the DLQI score will depend on other characteristics such as PASI score, marital status, education, and duration of disease.

If the patient's coping score is less than 171, the PASI score is above 17, and the patient is either married or single, the DLQI score is predicted to be about 20 , while if the patient is divorced or widowed, the DLQI score is predicted to be higher, up to 23 . This result suggests that, 
other things being equal, patients who experienced loss of a spouse or are divorced have lower quality of life than patients who are married or single. On the other hand, married patients with a coping score less than 171, a PASI score between 15 and 17, and duration of disease above 5.5 years have a predicted higher DLQI score (above 22), as opposed to single, divorced or widowed patients with a predicted lower DLQI score (up to 18). One can conclude that married patients with longer duration of disease have more trouble in fulfilling social responsibilities than single, divorced or widowed patients. With the same characteristics, if duration of disease is less than 5.5 years, the DLQI score is predicted to be about 17 regardless of marital status.

Following the same set of analyses, one can predict the DLQI score based on patient scores in different disease coping strategies. From Figure $2 \mathrm{~B}$ it can be deduced that the patients coping with disease using "Emotional and social support" (>12) and "Positivity and growth" ( $>10$ ) have a lower DLQI score and thus better quality of life. For the patients with "Emotional and social support" $(<12)$ the prediction of DLQI score depends on a combination of "Mental health", "Planning", and "Positivity and growth" strategies.

Regression analysis helps to identify patients' DLQI score based on overall profile characteristics and disease coping strategies. Based on regression results and selecting only statistically significant variables (having a $p$-value $<11 \%)$, regression equations are given: DLQI $=-0.08 \times$ Coping $+31.78 \times$ Elementary $+33.7 \times$ Higher + $32.05 \times$ High school; DLQI $=1.35 \times$ Mental health $+0.47 \times$ Positivity and growth.

Using regression equations, it is easy to calculate that patients with a total coping score less than 140 or patients with "Mental health" more than 16 will have a DLQI score above 21. This would fall into the category of patients whose quality of life is extremely affected by psoriasis. These patients require immediate attention and skills training for them to better cope with the disease.

In conclusion, the results of the study indicate that for patients with lower coping scores $(<140)$ the disease is most likely to have an extremely large effect on the quality of life as measured by the DLQI score (> 21). The same is true for the patients with underdeveloped disease coping strategies or a wrong mix of them. The results of the study indicate that psoriasis patients who suffer most considering their quality of life require more training in an efficient mix of coping strategies in the categories of "Emotional and social support", "Positivity and growth", "Mental health" and "Planning".

\section{Conflict of interest}

The authors declare no conflict of interest.

\section{References}

1. Raychaudhuri SP, Farber EM. The prevalence of psoriasis in the world. J Eur Acad Dermatol Venereol 2001; 15: 16-7.

2. Kouris A, Christodoulou C, Kontochristopoulos G, et al. Quality of life and psychological aspects in Greek patients with psoriasis: a cross-sectional study. An Bras Dermatol 2015; 90: 841-5.

3. Parrish L. Psoriasis: symptoms, treatments and its impact on quality of life. Br I Community Nurs 2012; 17: 524-8.

4. Palijan TZ, Kovacevic D, Vlastelica M, et al. Quality of life of persons suffering from schizophrenia, psoriasis and physical disabilities. Psychiatr Danub 2017; 29: 60-5.

5. Lakuta P, Marcinkiewicz K, Bergler-Czop B, Brzezinska-Wcislo L. How does stigma affects people with psoriasis? Adv Dermatol Allergol 2017; 34: 36-41.

6. Gelmetti C. Therapeutic moisturizers as adjuvant therapy for psoriasis patients. Am J Clin Dermatol 2009; 10: 7-12.

7. Picardi A, Pasquini P. Toward a biopsychosocial approach to skin disorders. Adv Psychosom Med 2007; 28: 109-26.

8. Mazzotti E, Mastroeni S, Lindau J, et al. Psychological distress and coping strategies in patients attending dermatology outpatient clinic. J Eur Acad Dermatol Venereol 2012; 26: 746-54.

9. Fortune DG, Richards HL, Main CJ, Griffiths CE. Patients strategies for coping with psoriasis. Clin Exp Dermatol 2002; 27: 177-84.

10. Leibovici V, Canetti L, Yahalomi S, et al. Well-being, psychopathology and coping strategies in psoriasis compared with atopic dermatitis: a controlled study. J Eur Acad Dermatol Venereol 2010; 24: 897-903.

11. Hrehorow E, Salomon J, Matusiak L, et al. Patients with psoriasis feel stigmatized. Acta Derm Venereol 2012; 92: 67-72.

12. Louden BA, Pearce DJ, Lang W, Feldman SR. A simplified psoriasis area severity index (SPASI) for rating psoriasis severity in clinic patients. Dermatol Online J 2004; 10: 7.

13. Pathirana D, Nast A, Ormerod AD, et al. On the development of the European S3 guidelines on the systemic treatment of psoriasis vulgaris: structures and challenges. J Eur Acad Dermatol Venereol 2010; 24: 1458-67.

14. Finlay AY. Current severe psoriasis and the rule of tens. $\mathrm{Br}$ J Dermatol 2005; 152: 861-7.

15. Finlay AY, Khan GK. Dermatology Life Quality index (DLQI) a simple practical measure for routine clinical use. Clin Exp Dermatol 1994; 19: 210-6.

16. Shankar V, Ghosh S, Ghosh K, Chaudhuri U. PASI and PQOL12 score in psoriasis: is there any correlation? Indian J Dermatol 2011; 56: 287-9.

17. Finlay AY, Khan GK. Dermatology Life Quality index (DLQI). Available at: Cardiff University Department of Dermatology website, http://www.cardiff.ac.uk/dermatology/quality-oflife/dermatology-quality-of-life-index-dlqi/ [Data Accessed: November 21, 2015].

18. Carver CS, Scheier MF, Weintraub JK. Assessing coping strategies: a theoretically based approach. J Pers Soc Psychol 1989; 56: 267-83. 